\title{
Importancia de la gestión de proyectos en la construcción civil de un teleférico
}

Importance of project management in the civil construction of an urban cable transport system

Jorge Antonio Molina Galván

jorgemolinag77@hotmail.com

Código ORCID: 0000-0002-0949-7450

\section{Universidad Privada Boliviana \\ La Paz - Bolivia}

4 Articulo aceptado en septiembre 2020 < Arbitrado en octubre 2020 < Publicado en enero 2021

La gestión de proyectos en la construcción civil y el análisis de casos reales cobraron importancia en las últimas décadas, así este estudio se propone analizar en retrospectiva la gestión del proyecto de construcción de la infraestructura de un teleférico, manifestando problemas y soluciones adoptadas durante su ejecución. Aplicando el método estudio de caso (Líneas Blanca y Celeste del Teleférico de La Paz, Bolivia), se investigó y evaluó la gestión del proyecto, siguiendo 4 pasos: descripción del proyecto, identificación de problemas, retro análisis, descripción de soluciones implementadas. Esta metodología permitió identificar 5 problemas trascendentales, relacionados con: modificación de la funcionalidad de operación, liberación de predios, cambios de trazado y modificación de la infraestructura e inestabilidad de taludes. Se concluye que frente a problemas de gran magnitud la gestión del proyecto fue crucial. Asimismo, resaltan lecciones aprendidas que son de gran utilidad para proyectos similares alrededor del mundo.

Palabras clave: Teleférico; Transporte por cable; Gestión de proyecto; Construcción civil

\section{Abstract}

Project management in civil construction and the analysis of real cases gained importance in recent decades. The purpose of the study is to retrospectively analyse the management of a cable car infrastructure construction project, showing problems and solutions adopted during its execution. Applying the case study method (Línea Blanca y Celeste of the cable car in La Paz, Bolivia), the project management was investigated and evaluated, following 4 steps: description of the project, identification of problems, back-analysis, description of implemented solutions. This methodology allowed the identification of 5 transcendental problems, related to: modification of the operation functionality, release of properties, changes of layout and modification of the infrastructure and slope instability. It is concluded that in the face of problems of great magnitude, the project management was crucial. They also highlight lessons learned that are especially useful for similar projects around the world.

Keywords: Cableway; Cable transport; Project management; civil construction 


\section{INTRODUCCIÓN}

La competitividad de un país está estrechamente relacionada con el grado de desarrollo de la infraestructura de transporte de sus ciudades, ya que ésta permite la correcta interconexión de los centros de producción, distribución y consumo y el traslado eficiente de población a sus centros de trabajo. Adicionalmente, la modernización de la infraestructura vial y de las distintas modalidades de transporte repercuten en la calidad de vida de la población, al disminuir tiempos de viaje, optimizar costos y garantizar el acceso a los servicios; así como reducir los niveles de congestionamiento, contaminación, accidentes y demoras, Figueroa (2005) \& Barbero (2016).

En este sentido, existen en deferentes países áreas urbanas que por la complejidad de condiciones particulares han demandado soluciones alternativas al transporte urbano tradicional. Este es el caso del transporte urbano en las ciudades bolivianas de La $\mathrm{Paz} y$ El Alto que ha sido constantemente estudiado y analizado a nivel estatal, debido a las condiciones críticas de congestionamiento, deterioro de vías y las deficiencias en los servicios provistos por los operadores tradicionales difícilmente regulados. Ante ello, el Sistema de Transporte por Cable (STC) surge en Bolivia como una solución ante las necesidades de transporte público de ambas ciudades, iniciando la construcción el año 2014, PNUD (2015).
Se presenta parte de la proposición teórica de que la gestión del proyecto tiene un papel fundamental para el cumplimiento de objetivos en la construcción de la infraestructura de obra civil de un sistema de transporte urbano por cable y se propone como objetivo realizar un retro análisis de la gestión del proyecto de construcción del teleférico en la ciudad de La Paz, Bolivia (Líneas Blanca y Celeste), manifestando la trascendencia de los problemas identificados y las soluciones adoptadas durante su ejecución.

El proyecto de construcción civil de este sistema es relevante para la comunidad científica debido a su dimensión y particularidades, aquí radica la necesidad de establecer un lineamiento de gestión técnico e ingenieril que permita materializar $y$ hacer posible la construcción de la infraestructura para la operación y funcionamiento comercial del teleférico en área urbana, con ello se puede garantizar la ejecución integral de las obras traducidas en estructuras capaces de sostener la movilidad de este sistema de transporte por cable. Cada uno de los aspectos de gestión del proyecto durante la construcción es importante para asegurar la conclusión del proyecto, certificación para la operación y puesta en marcha del teleférico. Así, la gestión de un proyecto de tal magnitud es un caso de estudio de gran interés para instituciones y profesionales en el área de ingeniería civil, ya que las lecciones aprendidas sirven para futuros proyectos de construcción al alrededor del mundo. 
En este sentido se ve la necesidad de conocer los antecedentes de proyectos similares, para tener un precedente de la importancia y complejidad que representa implementar un sistema de transporte por cable. Se presentan algunas experiencias de proyectos de construcción similares alrededor del mundo, el teleférico de Medellín, Colombia, se encuentra ligado a estaciones de metro, principalmente en zonas ubicadas en cerros y con mayor vulnerabilidad social. Actualmente cuentan con cuatro líneas de teleférico, las cuales llegan a transportar entre 1,200 hasta 3,000 personas por hora, por sentido. Aún existen detalles de capacidad por solucionar, pues en ciertas estaciones, los pasajeros tienen que esperar hasta 30 min para poder abordar, cuando el recorrido es de aproximadamente 15 minutos. Aun así, el teleférico es mejor opción que usar el bus, pues este tarda una hora, Rincón (2013).

El teleférico del Estado de México, en México, fue creado con el propósito de trasladar a los pasajeros de las zonas más marginadas a la zona urbana del Valle de México. El teleférico recorre una distancia de aproximadamente 5 $\mathrm{km}$ en 17 minutos, por tierra, lleva alrededor de $40 \mathrm{~min}$. Esta línea tiene capacidad para transportar hasta 6,000 pasajeros por hora y cada góndola alcanza una velocidad de hasta 18 $\mathrm{km} / \mathrm{h}$. Este teleférico ha sido diseñado para trabajar con energía solar, para reducir hasta 17,400 toneladas de CO2.
La línea está conectada con una estación de autobús, Tapia (2016).

Por su parte, el transporte por cable ubicado en Santiago (Chile), cuenta con 14 torres y 46 cabinas. Está diseñado para poder llevar bicicletas y carros especiales para personas con capacidades diferentes. Existe otro teleférico en el Cerro de San Cristóbal, pero éste se usa únicamente como medio de transporte para los turistas, Flores (2017). Este mismo tipo de transporte en Caracas, Venezuela, recorre una distancia de $4.8 \mathrm{~km}$ en 17 minutos y cuenta con 144 cabinas con capacidad de hasta 8 personas, Montoya (2017).

El teleférico de Brest, Francia, circula sobre el Río Penfeld que divide la ciudad de Brest en dos, posee capacidad para 60 personas por un trayecto de 420 metros que realiza en menos de tres minutos. Tiene dos especificidades: cuenta con dos cabinas que no se cruzan, sino que una discurre por encima de la otra, y una parte de los cristales se tinta cuando el aparato se acerca a viviendas para preservar la intimidad de los habitantes, Nuessgen (2015).

En Estados Unidos, en la ciudad de Nueva York, este mismo transporte toma menos de tres minutos de viaje, una distancia de 940 metros donde la cabina, que puede transportar hasta 110 pasajeros, se eleva a una altura máxima de 76 metros. Inaugurado en 1976 y remodelado en 2010, mueve anualmente más de dos millones de pasajeros, Suárez (2017). 
Y llegamos al teleférico de Bolivia, opera desde mayo de 2014, es el más largo del mundo, con una longitud de $36 \mathrm{~km}, 11$ estaciones y con capacidad de transporte promedio de 20,000 pasajeros cada hora (Fase 1: Líneas Roja, Amarilla y Verde), une a las ciudades de El Alto y La Paz. A inicios del año 2020 se inauguró la ampliación de siete nuevas líneas para integrar a barrios periféricos en la urbe paceña (Líneas Azul, Naranja, Blanca, Celeste, Morada, Café y Plateada).

\section{MÉTODO}

documentación fue revisada sistemáticamente en busca de detectar problemas que hubieran podido causar alteraciones al desarrollo del proyecto, afectando al logro de objetivos contractuales.

Los resultados que se muestran son fruto de esta revisión documental, con la cual se obtuvo una valoración técnica en retrospectiva. Presentada en cuatro pasos: 1) descripción del proyecto, 2) causas y problemas identificados, 3) evaluación y retro-análisis $\quad y, \quad 4)$ Soluciones implementadas.

Dada gran cantidad de información que se tuvo a disposición y que fue de vital importancia, este informe presenta la descripción de los documentos analizados y sintetiza de la gestión del proyecto según 5 macro actividades: 1) estudios y diseños (estudios TESA y complementarios), 2) ejecución, ingeniería del proyecto, 3) construcción, equipamiento y montaje, 4) puesta en marcha $y, 5)$ operación $y$ mantenimiento.
Se trata de una investigación con alcance descriptivo que aplica el método "estudio de caso", Martínez (2006). Siendo su unidad de análisis el proceso de ejecución del proyecto de construcción del teleférico en la ciudad de La Paz, Bolivia (Líneas Blanca y Celeste).

Para estudiar el proceso de ejecución del proyecto se aplicó la técnica de investigación y evaluación. Inicialmente se recogió información relevante referida al análisis de viabilidad, planificación, ejecución, seguimiento y control y cierre). Toda esta

A continuación, se identificaron cinco problemas de mayor relevancia, en cada uno de los casos se describió el suceso y se describieron las causas. Con relación a la evaluación y retroanálisis, siguiendo esta misma lógica, se procedió a evaluar por separado el papel de la gestión técnica del proyecto, valorando las posibles medidas y los factores a considerar. Finalmente, se presentaron las soluciones implementadas para cada problema.

\section{RESULTADOS}

\section{DESCRIPCIÓN DEL PROYECTO}

Se presenta la descripción del proyecto que fue caso de estudio (construcción de la infraestructura del sistema de transporte por cable en área urbana, líneas Blanca y Celeste).

Línea Blanca: se encuentra emplazada en la ciudad de La Paz, su infraestructura está dispuesta en dos secciones, 2.847 metros de longitud, distribuidas en 4 estaciones (San Jorge, 
Plaza Triangular, Busch y Plaza se movilizan a $5 \mathrm{~m} / \mathrm{s}$, con capacidad Villarroel), 26 torres y 131 cabinas, que de 3.000 Pasajeros-Hora/Sentido.

Tabla 1. Distribución de Estaciones y Torres en Línea Blanca

\begin{tabular}{|c|c|c|c|c|}
\hline Línea & Sección & Long. (m) & Estación & Torres \\
\hline \multirow{6}{*}{ LANCA } & \multirow{4}{*}{1} & \multirow{4}{*}{716,78} & Est. San Jorge (E-B2)* & \\
\hline & & & & T-B09 a T-B17 (8 torres) \\
\hline & & & Est. Triangular (E-B3) & \\
\hline & & & & T-B18 a T-B25 (8 torres) \\
\hline & \multirow{2}{*}{2} & \multirow{2}{*}{$2.130,46$} & Est. Busch (E-B4) & \\
\hline & & & Est. Villarroel (E-B5) & T-B26 a T-B35 (10 torres) \\
\hline
\end{tabular}


Línea Celeste: se encuentra emplazada en la ciudad de La Paz, su infraestructura está dispuesta en dos secciones, 2.595 metros de longitud, distribuidas en 4 estaciones (Libertador, San Jorge, Cancha Zapata y Prado), 26 torres y 155 cabinas, que se movilizan a $6 \mathrm{~m} / \mathrm{s}$, con capacidad de 4.000 PasajerosHora/Sentido.

Tabla 2. Distribución de Estaciones y Torres en Línea Celeste

\begin{tabular}{|c|c|c|c|c|}
\hline Línea & Sección & Long. (m) & Estación & Torres \\
\hline \multirow{6}{*}{ CELESTE } & \multirow{4}{*}{1} & \multirow{4}{*}{986,55} & Est. Libertador (E-Z1) & \\
\hline & & & & T-Z01 a T-ZO8 (8 torres) \\
\hline & & & Est. San Jorge (E-Z2) & \\
\hline & & & & T-ZO9 a T-Z17 (9 torres) \\
\hline & \multirow{2}{*}{2} & \multirow{2}{*}{$1.768,53$} & Est. Zapata (E-Z3) & \\
\hline & & & Est. Prado (E-Z4) & T-Z18 a T-Z26 (9 torres) \\
\hline
\end{tabular}

Para el estudio, el proceso general se sistematizó en 5 macro actividades (figura 1), el desglose es el siguiente: 1) Estudios y diseños, Estudios TESA y complementarios. Incluyen los estudios previos que corresponden a: Actualización del Estudio de identificación, topografía previa y el estudio de trazados. Además, se incluyen otros estudios complementarios. 2) Ejecución, ingeniería del proyecto. Contiene los componentes de obras civiles, sistemas electromecánicos y complementarios y obras complementarias. 3) Construcción, equipamiento y montaje. 4) Puesta en marcha y 5) Asistencia técnica / acompañamiento a operación y mantenimiento durante el funcionamiento comercial / operación comercial. 


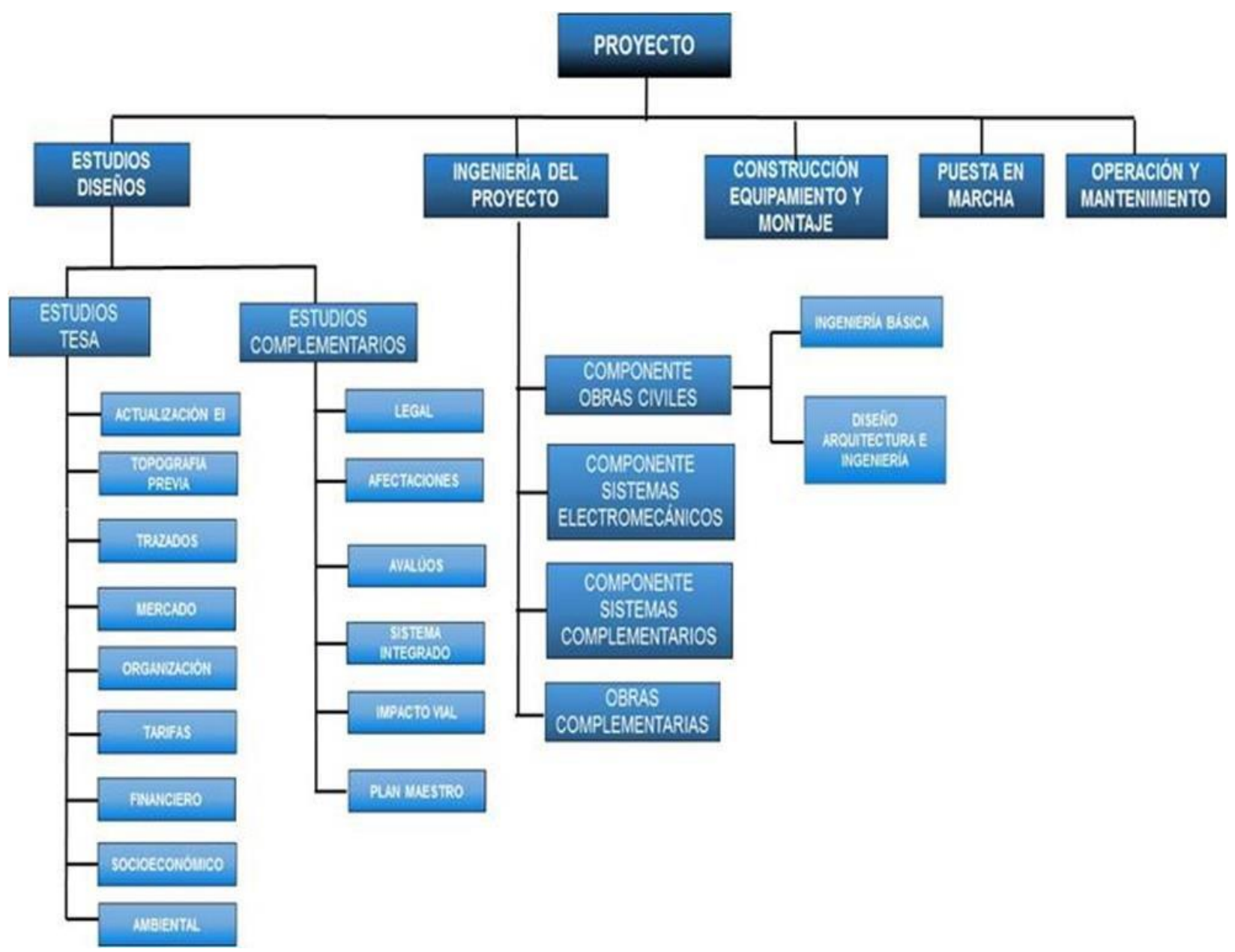

Figura 1. Proceso general del proyecto de construcción de infraestructura del sistema de transporte por cable en área urbana

A continuación, se presenta una síntesis de las actividades críticas del proyecto, se hizo énfasis en aquellas que implicaron una gestión activa en cuanto a la ejecución, control y evaluación.

Tabla 3. Síntesis de la gestión del proyecto según 5 macro actividades

\begin{tabular}{clll}
\hline $\begin{array}{c}\text { Macro } \\
\text { Actividades }\end{array}$ & \multicolumn{2}{c}{ Descripción de la actividad } & \multicolumn{2}{c}{ Gestión de la actividad } \\
\hline Estudios TESA & Estudios Técnico Socioeconómico y & Para la correcta gestión del \\
& Ambiente: fueron el soporte de la proyecto, dadas las dimensiones de \\
& ejecución del proyecto. Su meta este, se desarrolló una metodología \\
& principal fue demostrar la sostenibilidad de aprobación de documentos. \\
& del proyecto, determinando en este La metodología fue aplicada a \\
& caso la tarifa comercial con la cual los todos los estudios que constituyen el \\
& usuarios podrán hacer uso del medio de & TESA, logrando un orden adecuado \\
& transporte por cable. & a lo largo de la ejecución.
\end{tabular}


Continuación Tabla 3. Síntesis de la gestión del proyecto según 5 macro actividades

Macro
Actividades Descripción de la actividad Gestión de la actividad

Estudios Estudios emergentes como

complementarios consecuencia de la ejecución de las actividades de obra que permiten alcanzar la funcionalidad de infraestructura civil contemplado en desarrollo del Proyecto.

Ingeniería del proyecto

Construcción, equipamiento y montaje
Puesta en marca Pruebas de funcionalidad del sistema de transporte por cable teniendo como meta final una certificación internacional que permite el abordaje de los pasajeros de manera segura.

La gestión del proyecto debe garantizar el correcto funcionamiento durante la vida útil de la infraestructura, para eso la supervisión en todas las etapas previas es primordial.
La gestión implica asegurarse que estos estudios sean considerados conforme el avance del Proyecto. Por esto fue crucial hacer un seguimiento continuo.

La gestión implica asegurarse que los estudios sean considerados conforme el avance del Proyecto. Por esto fue crucial hacer un seguimiento continuo.

Fue de vital importancia mantener un buen relacionamiento entre las Partes responsables: Contratista, Supervisión y Fiscalización.

Por otra parte, fue importante cuidar las relaciones con otros involucrados: Embajada de Brasil, vecinos afectados, entre otros.

El cumplimiento de los plazos de entrega estuvo en riesgo en reiteradas ocasiones, se tomaron acciones inmediatas para evitar afectar la ruta crítica. Si embargo hubo dos ocasiones en que frente a factores externos y no se logró cumplir los tiempos programados. Se procesión entonces a respaldas lo motivos y se tramitó el Certificado de Constancia de Existencia de Impedimento debido a Fuerza Mayor.

Se dio la debida importancia al seguimiento a las pruebas, siendo vital el control del cronograma.

Se gestionó y supervisó la implementación del programa "aprender haciendo", impartido por el Contratista y con la correspondiente Supervisión.

La gestión del proyecto previó en las etapas anteriores el correcto funcionamiento comercial.

Por ejemplo, se evaluó cada 3 meses el comportamiento de los taludes en la atapa de construcción. 
Durante la ejecución del proyecto existieron eventualidades que generaron modificaciones en cuanto al alcance, metodología y procedimientos constructivos, implicando con ello redistribución de los recursos económicos, humanos, materiales, equipo y maquinaria y repercutiendo a la vez en los plazos de cronogramas de ejecución para su puesta en marcha y funcionamiento comercial.

Se refleja las lecciones aprendidas y oportunidades de mejora durante la ejecución de la obra. Sirva esto como antecedente para futuros proyectos similares en el área del transporte y movilidad de pasajeros.

\section{CAUSAS Y IDENTIFICADOS \\ PROBLEMAS}

Por diferentes razones de índole técnico, social y/o legal, surgieron problemas que trascendieron más allá de los problemas tradicionales de construcción. Los problemas de mayor relevancia son:

\section{Modificación de la funcionalidad de operación de la Estación E-B2}

El contrato de obra establecía inicialmente que la Línea Blanca estaba concebida a partir de un trazado que conectaba las Líneas Amarilla y Verde en E-B1 y concluía en la E-B5.

A partir de una reevaluación de la demanda de usuarios de la Línea Celeste, esta requería iniciar en E-B1, lo cual afectó directamente a la Línea Blanca. Implicaría al mismo tiempo una modificación en el diseño de la infraestructura de soporte y también en la fabricación del sistema electromecánico, considerando que ya se disponía de un cronograma inicial de ejecución de obra aprobado, generando reducción y eliminación del trazado entre E-B1 y E-B2 originalmente previstas.

\section{Escaleras mecánicas en E-B2 para conexión con avenida}

Según contrato de obra se tenía prevista la construcción de escaleras mecánicas en la E-B2 que permitían la conexión entre el edificio de E-B2 y el pasillo de conexión dispuesto a nivel de la avenida, logrando así superar el desnivel y garantizar un tránsito seguro y cómodo para los usuarios.

Realizado una reevaluación de este tipo de conexión, se identificó que las características topográficas del lugar no permitían implantar de manera adecuada las escaleras mecánicas, por otra parte, el tiempo que requería el usuario para llegar a la la avenida (aprox. $10 \mathrm{~min}$ ) era excesivo, debido a la longitud que demandaban las escaleras para vencer el desnivel del terreno natural existente.

\section{Liberación de predios para cimentaciones del Edificio de Ascensores en E-B2}

A partir del contrato, se dispuso la implantación de un edificio de ascensores en E-B2 que permitía la conexión entre la E-B2 y la avenida.

Al momento de iniciar los trabajos previos de prospección geotécnica, se 
concretó la inaccesibilidad a predios que pertenecían a terceros, debido a que existían intereses económicos y personales por parte de los copropietarios. Esto impidió que el proceso de liberación se desarrolle de manera normal, impidiendo iniciar las tareas de prospección geotécnica como de obras a posterior

El impedimento para ingresar a los predios dispuestos en el talud de la parte posterior de E-B2 afectó de manera directa al avance normal de la obra, generando atrasos de consideración en el cronograma de ejecución de la Línea Blanca. Se tuvo atrasos en la consolidación del diseño definitivo de la estructura como consecuencia de no contar con los estudios completos de prospección geotécnica de la zona.

\section{Cambio del trazado y modificación de la infraestructura de la Línea Celeste}

La Línea Celeste (Sur) debía tener un trazado que iniciaba en la Línea Verde. A partir de la actualización de los estudios de la líneas de demanda del STC realizada por el contratista, luego de recibir la orden de proceder, se detectó una demanda de usuarios desde la zona sur al centro de la ciudad de La Paz, por lo que se reevaluó la posibilidad de implementar una línea de teleférico que permita el transporte de pasajeros desde la zona sur hasta el centro paceño.

La implementación de esta nueva línea implicaba un incremento al monto del Proyecto, situación que era poco factible debido al elevado costo que representaba en aquel momento, por otro lado, significaba una modificación de consideración a la infraestructura de soporte, además de los diseños y fabricación de los equipos del sistema electromecánico, no solo de esta nueva línea sino de aquellas que debieran conectarse (Línea Blanca).

\section{Estabilidad de taludes $y$ cimentaciones de E-Z2}

A partir del contrato se dispuso la implantación de la Línea Celeste (Centro), que tenía prevista la construcción de E-B1, E-B2, E-Z3 y E-Z4. A consecuencia de los diseños del sistema electromecánico y de funcionalidad con respecto a la RIM de teleféricos, fue conveniente emplazar la estación E-Z2 sobre la E-B2.

Al momento de realizar los trabajos preliminares de prospección geotécnica se evidenció la disposición de material de relleno (no propio del lugar) en los estratos inferiores y talud del lugar de emplazamiento de la infraestructura de la estación, identificando por debajo del nivel de terreno el embovedado del Río Choqueyapu y en el talud la presencia de material de relleno saturado y con niveles freáticos variables.

Estos factores repercutían en una evaluación particular y una readecuación del diseño de la infraestructura de soporte del sistema electromecánico $y$, por tanto, demandaban mayor tiempo de ejecución de la obra que vulneraba el 
cumplimiento de los plazos establecidos en el cronograma.

\section{EVALUACIÓN Y RETRO - ANÁLISIS}

\section{Modificación de la funcionalidad de operación de E- B2}

En su momento se dispuso de los siguientes documentos contractuales: contrato de obra, cronograma inicial de obra, DBCD y TDR, propuesta técnica aceptada.

Las causas del problema fueron: 1) Necesidad de implementar la nueva Línea Celeste (Centro). 2) Se tuvo que evitar el sobrevuelo sobre predios de la embajada del Brasil. El trazado original de la Línea Blanca hubiera demandado una serie de gestiones gubernamentales con la Embajada Brasileña para lograr la aprobación del sobrevuelo de las cabinas sobre esta. El cambio de trazo repercutía en el alineamiento del trazado definitivo de esta Línea y con ello nuevos estudios y diseños, generando así un atraso en el avance de las obras y cumplimiento de plazos contractuales.

En vista de estos argumentos de carácter técnico y legal se analizaron los siguientes aspectos antes de adoptar solución definitiva: Primero, para la implementación de la nueva Línea Celeste (Centro) se valoró los costos económicos que efectivamente ascendían a montos muy por encima del valor que el contrato permite por cualquier modificación (10\%). Sin embargo, el problema no radica únicamente en ello, sino en la disponibilidad del recurso y las gestiones y cumplimiento de procedimientos para disponer del monto adicional. Asimismo, ante la prioridad de insertar la nueva Línea Celeste (Centro), los alcances y objetivos de la Línea Blanca fueron cuestionados y se analizó su reducción, se buscó un escenario en el que fuera posible la conectividad de esta línea con la nueva y con la RIM de teleféricos. Por último, se analizó el punto de intersección físico entre las Líneas Blanca y Celeste, siendo este E-B2. La ubicación de este punto emplazado en una topografía compleja, con características geológicas variables por la configuración principalmente de los taludes, implicó la posibilidad de reestablecer la directriz inicial de construcción de las obras y a la vez la previsión para la fabricación de piezas del sistema electromecánico.

\section{Escaleras mecánicas en E-B2}

En su momento se dispuso de los siguientes documentos contractuales: contrato de obra, cronograma inicial de obra, DBCD y TDR, propuesta técnica aceptada.

Las causas detectadas fueron dos: a) El tiempo de salida del usuario desde la E-B2 hasta la avenida era relativamente extenso, se manejaba un tiempo aproximado de 10 a 12 minutos haciendo uso de las escaleras mecánicas. b) Complejidad en la materialización de la infraestructura de soporte de las escaleras. Por la configuración topográfica, la longitud de las escaleras requería una serie de 
estructuras que dificultaría el posterior mantenimiento de los equipos.

En vista de estos argumentos se analizaron los siguientes aspectos antes de adoptar solución: a) Uno de los propósitos de la implementación de los teleféricos en área urbana es el de acortar distancias y otorgar un viaje seguro, eficiente y en el menor tiempo posible. La Línea Blanca inicialmente estimaba un tiempo de viaje (de E-B1 hasta E-B5) de 20 minutos. Con el trazado modificado (de E-B2 hasta E-B5) el tiempo reducía a 13 minutos, sin embargo, a este tiempo se añadió 11 minutos, que es el tiempo que demoraría el usuario en recorrer a través de las escaleras mecánicas desde el desembarque de la cabina en estación E-B2 hasta llegar a la avenida. b) El tiempo efectivo de viaje de la Línea Blanca resultaba ser 24 minutos, de los cuales el $46 \%$ representaba el viaje a través de las escaleras, situación poco aceptable. Por tanto, es aquí donde radica la reevaluación de la implementación de la estructura prevista en los TDR. C) El costo económico de una nueva estructura que permita reducir el tiempo de viaje efectivo, implicaría un incremento al monto del contrato, esto limitaba el tipo de solución a adoptar. d) Era necesario contar con un análisis preliminar de los tiempos efectivos de viaje desde en ingreso del usuario a E-B2 hasta la salida de este, el análisis debe ser cuantificado en los estudios preliminares que forman parte de los documentos de licitación del proyecto, a fin de evitar cambios de consideración durante la ejecución de las obras.

\section{Liberación de predios para cimentaciones del edificio de ascensores estación E-B2}

En su momento se dispuso de los siguientes documentos contractuales: contrato de obra, cronograma aprobado del CM 1, DBCD y TDR, propuesta técnica aceptada.

Como principales causas se entienden: 1) Liberación tardía de terreno, debido a intereses personales de tipo económico por parte dueños del predio requerido para el desplazamiento de equipo de prospección geotécnica y posterior emplazamiento del edificio de ascensores como estructura de conexión entre la E-B2 y la avenida. 2) identificación y notificación tardía de los predios a ser expropiados para la implantación del edificio de ascensores.

Se analizaron los siguientes aspectos antes de adoptar solución: a) A la firma del CM 1, se establecen nuevos plazos contractuales para el FCOM LB. Se requirió la ampliación del tiempo estipulado inicialmente para la construcción de E-B2, ya que la ejecución de un edificio de ascensores de $42 \mathrm{~m}$ de altura (13 pisos), una pasarela y pasillo de conexión de longitud aproximada de 105 m hasta la avenida, representaba un volumen importante de obra en un solo frente de trabajo, como consecuencia de las características desfavorables de los estratos geológicos, topografía 
accidentada y accesibilidad al lugar (liberación tardía de predios). b) Si bien, el CM 1 estima un plazo ejecución conforme a los rendimientos que son usuales en estructuras similares, la liberación tardía del predio para la ejecución de los trabajos fue repercutió sobre el normal avance del cronograma de obra modificado. c) Una vez que fue posible acceder al predio liberado, se evidenció, a partir de los estudios de prospección geotécnica, condiciones desfavorables para la implantación del edificio de ascensores. Por lo que la importancia de disponer de los estudios básicos jugó un papel fundamental para la elaboración de los diseños preliminares y definitivos.

Para el problema descrito, la liberación tardía afectó directamente a la ruta crítica para el FCOM de la Línea Blanca, debido a que se generó un atraso en la ejecución del edificio de ascensores y con ello se impidió la llegada de los usuarios a la avenida como destino final de viaje.

\section{Cambio del trazado y modificación de la infraestructura de la Línea Celeste}

En su momento se dispuso de los siguientes documentos contractuales: contrato de obra, DBCD y TDR, propuesta técnica aceptada.

Las causas del problema fueron: 1) A partir de la actualización de los estudios de demanda de los TDR, se detectó la conveniencia de implementar una nueva línea de teleférico que permita la conexión entre la zona Sur y el Centro de la ciudad de La Paz, 2) Factibilidad técnica y económica, como consecuencia de una mejor redistribución de los recursos económicos asignados al Proyecto de teleféricos en su Fase 2.

Se consideró importante analizar tres argumentos antes de proceder: a) Suprimir la Línea Celeste (Sur) ya prevista en los estudios y documentos de licitación, implicaba la readecuación de algunos alcances del proyecto, que permitía abrir un nuevo escenario de socialización y adquisición de la licencia ambiental del nuevo trazado y justificación de la eliminación del trazado original. b) La actualización de los estudios de demanda de pasajeros a través de las encuestas de origen destino, representa un documento indispensable de elaboración prioritaria al momento de la licitación del proyecto. Este documento ahorrará tiempos de reevaluaciones y actualizaciones en la etapa de construcción, identificando de manera temprana posibles mejoras para el sistema de transporte integrado. c) La redistribución de los recursos económicos permite analizar los escenarios de mejoras al proyecto, de manera integral con las líneas componentes de la RIM. Estos escenarios permitirán optimizar diseños de manera eficiente y a la vez generar mejoras en beneficio de los usuarios.

\section{Estabilidad de taludes $y$ cimentaciones de la Estación E-Z2}

Para el análisis se dispuso de los siguientes documentos contractuales: 
contrato de obra, cronograma aprobado del CM 1, DBCD y TDR, propuesta técnica aceptada.

Las causas identificadas fueron dos: 1) Disposición de información técnica insuficiente que no permitió identificar de manera clara y oportuna elementos estructurales antiguos, en este caso la existencia del embovedado del Río Choqueyapu. 2) Un evento externo de fuerza mayor durante la ejecución de los trabajos, el deslizamiento de un talud que delimitaba el área de intervención de la Estación San Jorge. Este aspecto afectó negativamente a la ejecución de esta estación, en el sentido que demandaría la reevaluación de una solución ingenieril para la estabilización del talud.

En vista de estos argumentos se analizaron los siguientes tres factores antes de adoptar solución: a) La existencia del embovedado del Río Choqueyapu por debajo de lugar de emplazamiento de la estación E-B2 representaba un obstáculo para el avance normal de las obras, siguiendo los diseños tradicionales de este tipo de estructuras de soporte. Por lo que esto implicaba evaluar y analizar el nuevo escenario y con ello las soluciones ingenieriles adecuadas. b) Es importante destacar que la información técnica sobre el alineamiento y emplazamiento del embovedado debe ser clara y precisa, situación que no fue así, debido a la carencia de información por parte del GAMLP. Se evidencia la importancia de tener los planos As-Built de toda obra ejecutada a fin de que en un futuro sean útiles para los posibles proyectos que se ejecuten. c) La prospección geotécnica en taludes tiene su complejidad en cuanto a la movilización de los equipos necesarios, para el caso de estudio un equipo rotativo de perforación, sin embargo, lograr el desarrollo con éxito permite identificar posibles riesgos, además de una caracterización precisa de la geología.

\section{SOLUCIONES IMPLEMENTADAS}

Se presentan las soluciones implementadas en relación con los problemas estudiados en el caso de estudio:

\section{Modificación de la funcionalidad de operación de E- B2}

E-B2 cambió de ser una estación intermedia a una estación Terminal Motriz con la construcción de un garaje de cabinas (cap. 47 cabinas) y un edificio de ascensores (con 6-8 ascensores de cap. 18 personas.

Esta modificación definitiva respondió a la reducción del trazado original de la Línea Blanca en el tramo comprendido entre E-B1 y E-B2.

Como parte de la solución se ejecutaron obras complementarias: intercambiador modal para transporte público (Buses Puma-Katari, taxis y minibuses), estabilidad de taludes colindantes en la parte posterior de la estación y accesos vehiculares para trabajos de mantenimiento de la infraestructura. 
Escaleras mecánicas en estación EB2 para conexión avenida

Como solución definitiva se ejecutó la construcción del edificio de ascensores de $42 \mathrm{~m}$ de altura y 6 ascensores de capacidad para 18 personas cada uno. Esta estructura permitió reducir el tiempo efectivo de los usuarios logrando un tiempo de $15 \mathrm{~min}$, por otro lado, garantizaba un tránsito seguro y confortable para los usuarios, sin dejar de lado una mejora en cuanto a la accesibilidad del personal técnico y equipo para trabajos de mantenimiento del sistema e infraestructura civil.

\section{Liberación de predios para cimentaciones del edificio de ascensores estación E-B2}

La liberación tardía de los predios afectó directamente a la ruta crítica del cronograma de ejecución de la Línea Blanca. Como solución a este conflicto a causa de un tercero y condiciones geológicas desfavorables, se emitió por parte de la supervisión un certificado de "constancia de existencia de impedimento debido a fuerza mayor" a fin de respaldar los tiempos constructivos para la conclusión de los trabajos.

Con el fin de optimizar y evitar plazos extensos que demoren mucho más el FCOM LB, se modificó la metodología y proceso constructivos del edificio de ascensores, con el uso de encofrados metálicos prefabricados para la ejecución de vigas y columnas, y al mismo tiempo con la construcción de estructuras mixtas de $\mathrm{H}^{\circ} \mathrm{A}^{\circ}$ con perfiles metálicos en los niveles últimos del edificio.

\section{Cambio del trazado y modificación} de la infraestructura de la Línea Celeste

Se adoptó el nuevo trazado de la Línea Celeste (Centro) que iniciaba en E-B1 (Líneas Amarilla y Verde), pasaba por E-BA, E-Z3 y concluía en E-Z4.

El cambio de trazado implicó la construcción de 4 estaciones y 26 torres, con 155 cabinas en circulación y una capacidad del sistema de 4000 pasajeros/hr. El tiempo de recorrido de la línea es de 14 minutos, siendo esta línea una de las más rápidas por la velocidad que permite el sistema de 6 $\mathrm{m} / \mathrm{s}$.

\section{Estabilidad de taludes $y$ cimentaciones E-Z2}

Como solución al problema en la EZ2, se implementó un sistema de pilotaje para el soporte de la obra civil funcional (mástiles y torres), edificio de estación y obras de contención del talud. Los pilotes de hormigón armado han alcanzado una profundidad media de $20 \mathrm{~m}, 0,90 \mathrm{~m}$ de diámetro haciendo un total de 127 pilotes para cimentación de las estructuras mencionadas.

Este sistema de pilotaje garantizó la estabilidad del sistema y del edificio, sin que ello afecte a la estructura ya existente del embovedado del río Choqueyapu, por lo que 
la solución ingenieril ha sido la más idónea y adecuada para las condiciones existentes del lugar.

La implementación de esta solución estructural representó una modificación al plazo de entrega para FCOM LZ, por lo que fue necesario respaldar el mismo con la elaboración de un certificado de "constancia de existencia de impedimento debido a fuerza mayor" según las condiciones vigentes del contrato.

\section{CONCLUSIONES}

El método de caso de estudio, acompañado de la técnica de investigación y evaluación, permitieron reflejar los aspectos técnicos necesarios para la ejecución del proyecto, de manera ordenada se estudió las etapas evolutivas y de construcción, alcanzando la finalización del proyecto con la puesta en servicio para los usuarios. Se evaluó que la gestión del proyecto implicó un adecuado seguimiento, control y supervisión que, a la vez, permitieron su la correcta ejecución. De esta manera, quedó demostrara la proposición teórica y se afirma que la gestión de proyecto tiene un papel fundamental para el cumplimiento de las exigencias del cliente, en este caso la entidad contratante EETC - MT.

Se logró reflejar la gestión técnica con un adecuado seguimiento, control y supervisión para la construcción de la infraestructura del sistema de transporte por cable, con base al caso de estudio de las Líneas de teleférico Blanca y
Celeste, las cuales forman parte de la Red Integral Metropolitana (RIM) emplazada en la ciudad de La Paz. Se demuestra que la ejecución ordenada y secuencial de las actividades bajo el cumplimiento de las especificaciones técnicas permite alcanzar y dar cumplimiento al objeto principal del contrato. Asimismo, otorgar al usuario un sistema de transporte urbano eficiente, cómodo y seguro en un escenario complejo como es la ciudad de La Paz.

El análisis de la gestión técnica realizada en el presente caso es un referente para proyectos similares, coadyuvando en la construcción y puesta en servicio de teleféricos en el mundo, ya que estos representan en nuestro medio un impacto tecnológico, no solo por la infraestructura moderna implantada para funcionamiento del sistema electromecánico, sino también porque otorga al usuario un medio de transporte urbano eficiente, cómodo, seguro y amigable con el medio ambiente.

Se enfatizó el componente de obras civiles con los aspectos técnicos vitales y necesarios que de manera ordenada muestran las etapas constructivas hasta su culminación con la puesta en servicio para los usuarios, La gestión del proyecto implicó asegurarse que los estudios TESA y los estudios complementarios sean considerados permanentemente a lo largo del avance del Proyecto.

La construcción ordenada y según cronograma de obra permitió dar paso al montaje de las piezas 
electromecánicas, etapa en la cual se tuvieron mayores exigencias de control conforme a lo establecido por el compendio normativo. Esta etapa concluye con la realización de pruebas de funcionamiento al sistema con la verificación de una brigada técnica especializada de tipo internacional (TÜV SÜD Industrie Service $\mathrm{GmbH}$ ), la cual fue la única autorizada para brindar la Certificación Internacional para Operación del Sistema.

Durante el avance de los trabajos, una de las preocupaciones mayores fue el inicio de operaciones del sistema para funcionamiento comercial, debido a que esto significa en primer lugar, un cumplimiento contractual existente, otorgar una alternativa de transporte eficiente, seguro y confortable a la ciudadanía y finalmente ingresos económicos a la empresa estatal. Sin embargo, las fechas fueron modificadas en la etapa constructiva, como consecuencia de implementar mejoras al Sistema de Transporte por Cable. Es importante analizar que se hubiera podido evitar la demora si es que se contaba con los estudios complementarios de manera oportuna.

Se identificaron los problemas y las soluciones adoptadas como consecuencia de la ejecución del caso de estudio, aquellos de mayor relevancia para el proyecto fueron: necesidad de escaleras mecánicas no previstas en estudios TESA Y complementarios, impedimentos externos para liberación de predios para el edificio de ascensores en la estación
E-B2, trazado inicial línea blanca y celeste, estabilidad de taludes en estación E-Z2. El análisis de las soluciones implementadas refleja la importancia de la gestión técnica con un adecuado seguimiento, control y supervisión para la construcción de la infraestructura del sistema de transporte por cable.

Para la elaboración de los diferentes estudios y diseños que ha permitido la ejecución de las líneas, se ha dispuesto la elaboración de un compendio normativo, este instrumento es la base técnica sobre la cual se enmarcó el desarrollo de las actividades contempladas en el cronograma de obra. En él se encuentran plasmadas las normativas aplicadas, reglamentos y documentos técnicos que han garantizado dar cumplimiento a las especificaciones técnicas y términos de referencia exigidos por el cliente (EETC MT). Para ello se ha valido de los siguientes criterios de normativa a aplicar: norma local normativa internacional consulta internacional.

\section{REFERENCIAS BIBLIOGRÁFICAS}

Barbero, J. (2006). Transporte urbano. Giugale, MM, Fretes-Cibils, V. and JL Newman

EETC. Empresa Estatal de Transporte por Cable (2019)., Estudio Técnico, Económico, Social y Ambiental STC La Paz - El Alto. Bolivia

Figueroa, O. (2005). Transporte urbano y globalización: Políticas y efectos en América Latina. EURE. Chile

Flores, M. (2017). Desarrollo de las bases de un modelo de mantenimiento aplicado a sistema de transporte por cable tipo teleférico en Chile 
Martínez, P. (2006). El método de estudio de caso estrategia metodológica de la investigación científica. Pensamiento y Gestión, No 20 ISSN 1657-6276. España

Montoya, H., \& Coromoto, J. (2017). Anteproyecto arquitectónico y paisajístico de la estación San José, del sistema teleférico del litoral, Caracas-distrito Capital. Venezuela

Nuessgen, M. (2015). Urban Ropeways in Europe. Creating Opportunities in urban development. European Institute for Sustainable Transport

PNUD PROGRAMA DE NACIONES UNIDAS PARA EL DESARROLLO (2015), El nuevo Rostrode Bolivia: Transformación Social y
Metropolización. Informe Nacional sobre Desarrollo Humano (2015)

Rincón, E. \& Ordosgoitia, I. (2013). Claves del Éxito en Teleféricos y su Articulación con Planes de Desarrollo Urbano Integral: Metrocables Medellín. Estudios de Transporte Suárez-Alemán, A., \& Serebrisky, T. (2017). ¿̇Los teleféricos como alternativa de transporte urbano?: Ahorros de tiempo en el sistema de teleférico urbano más grande del mundo: La Paz-El Alto (Bolivia). Bolivia Tapia, V. (2016). Eficiencia Sectorial en la Administración Pública del Estado de México. México 